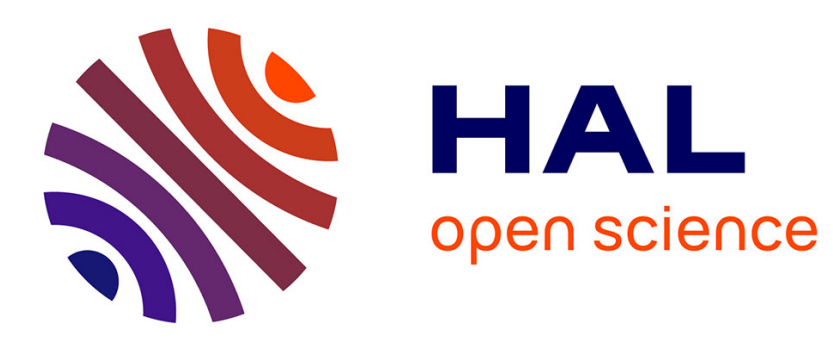

\title{
Theoretical study of lithium niobate slab waveguides for integrated optics applications
}

\author{
G.W. Burr, S. Diziain, M.-P. Bernal
}

\section{To cite this version:}

G.W. Burr, S. Diziain, M.-P. Bernal. Theoretical study of lithium niobate slab waveguides for integrated optics applications. Optical Materials, 2009, 31 (10), pp.1492-1497. 10.1016/j.optmat.2009.02.013 . hal-00370152

\section{HAL Id: hal-00370152 \\ https://hal.science/hal-00370152}

Submitted on 3 May 2021

HAL is a multi-disciplinary open access archive for the deposit and dissemination of scientific research documents, whether they are published or not. The documents may come from teaching and research institutions in France or abroad, or from public or private research centers.
L'archive ouverte pluridisciplinaire HAL, est destinée au dépôt et à la diffusion de documents scientifiques de niveau recherche, publiés ou non, émanant des établissements d'enseignement et de recherche français ou étrangers, des laboratoires publics ou privés. 


\title{
Theoretical study of lithium niobate slab waveguides for integrated optics applications
}

\author{
G.W. Burr ${ }^{\text {a }}$, S. Diziain ${ }^{\text {b,* }}$, M.-P. Bernal ${ }^{\text {b }}$ \\ a IBM Almaden Research Center, 650 Harry Road, San Jose, California 95120, USA \\ ${ }^{\mathrm{b}}$ Institut FEMTO-ST, Département d'Optique P.-M. Duffieux, CNRS UMR 6174, Université de Franche-Comté, 16 route de Gray, 25030 Besançon cedex, France
}

We report on the theoretical study of lithium niobate slab and wire waveguides with different kinds of cladding (silicon dioxide, sapphire and air). The mode propagation, the light confinement and radiation losses are simulated using a software based on a beam propagation method. We propose from those results lithium niobate waveguide geometries for optical integrated applications.

\section{Introduction}

Because of its excellent optical transparency in the visible and infrared and its remarkable electrooptic, ferroelectric and nonlinear optical properties, lithium niobate (LN) is an ideal material for versatile applications in photonics and optoelectronics [1]. All devices are commonly done in bulk but the use of LN thin films will be of great interest for miniaturization and integrated optics. Thin layers of LN are, however, very difficult to do. The most commonly methods employed to fabricate single-crystal LN thin films with bulklike properties are polishing or crystal ion slicing (CIS) [2-4]. With such techniques the periodicity of ferroelectric domains [5] as well as the nonlinear optical [6] or electro-optic modulation properties [7,8] are preserved in single-crystal LN thin films. Free-standing LN films find applications in wide-free-spectralrange Fabry-Perot optical filters [9], in second-harmonic-generation [5] but they are also suitable for hybrid optical integration as, for example, half-wave plates [10]. Long-range surface plasmon waveguides can also be fabricated by direct wafer bonding between thin metal layers and single-crystal LN thin films [11]. In this case, the electro-optic effect of LN can be used to tune the cutoff frequency of the waveguides. High-performance devices such as low-tunable TE/TM converters [12] or electro-optically tunable

\footnotetext{
* Corresponding author. Fax: +33 381666423.

E-mail address: severine.diziain@univ-fcomte.fr (S. Diziain).
}

second-harmonic-generation gratings [13] benefit from the high tuning per volt due to an improved overlap integral in the thin LN films. Another advantage of single-crystal LN thin films is the possibility to use them as slab waveguides. It has already been demonstrated that they allow terahertz wave generation and propagation [14]. LN slab waveguides would also be a good alternative to gradient index LN waveguides in order to obtain the light vertical confinement for photonic crystal (PC) devices. In fact, tunable LN PCs are usually composed of air holes etched by focused ion beam (FIB) in annealed proton exchange (APE) waveguides [15]. This fabrication method is however responsible for out-of-plane losses due to the conical shape and the finite depth of the holes combined with the weak confinement of the guided mode [16]. Etching holes in LN slab waveguides so that they go through the entire guided mode would limit those loses. In spite of the obvious interest of LN slabs for integrated optics, there is no theoretical study on the optical behaviour of such layers with respect to the material cladding to be used and the needed thickness for monomodal propagation.

In this paper, we focus on two kinds of smart guides, respectively called symmetric slab waveguides and asymmetric wire waveguides. The first geometry consists of a single-crystal LN thin film sandwiched between two layers of the same nature that can be either air, sapphire or silicon dioxide $\left(\mathrm{SiO}_{2}\right)$. The second type of smart guide is composed of a LN ridge deposited on two kinds of substrates (sapphire and $\mathrm{SiO}_{2}$ ). The choice of these layers arises 
from their refractive indices that are suitable for fabricating LN slab waveguides and from the possibility to bond them with LN films by wafer bonding or plasma-enhanced chemical vapor deposition (PECVD) $[8,17]$. For each structure, we are interested in the guided modes, their confinement and the possible coupling effects between guided modes and slab modes that could cause losses. For these purposes, the effective indices of each device are calculated as a function of geometric parameters (the height of the LN layer

\section{Z-cut}
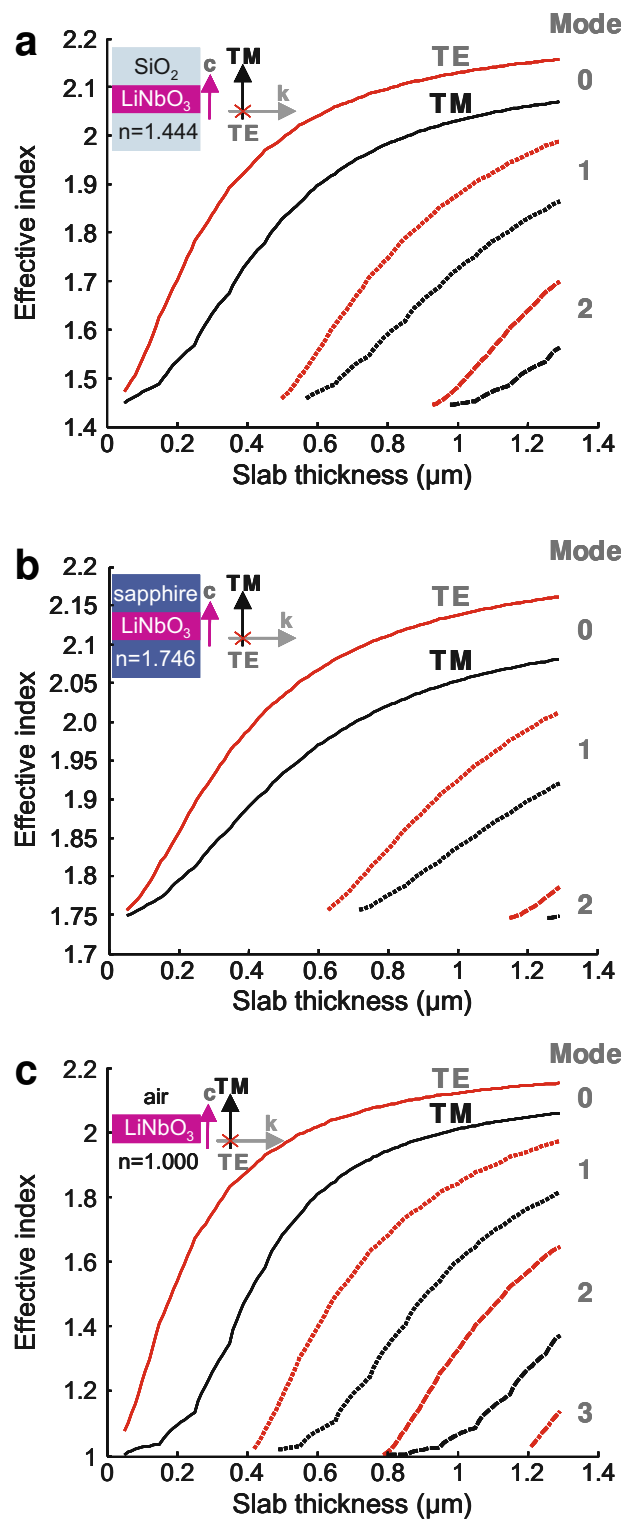

\section{X-cut}
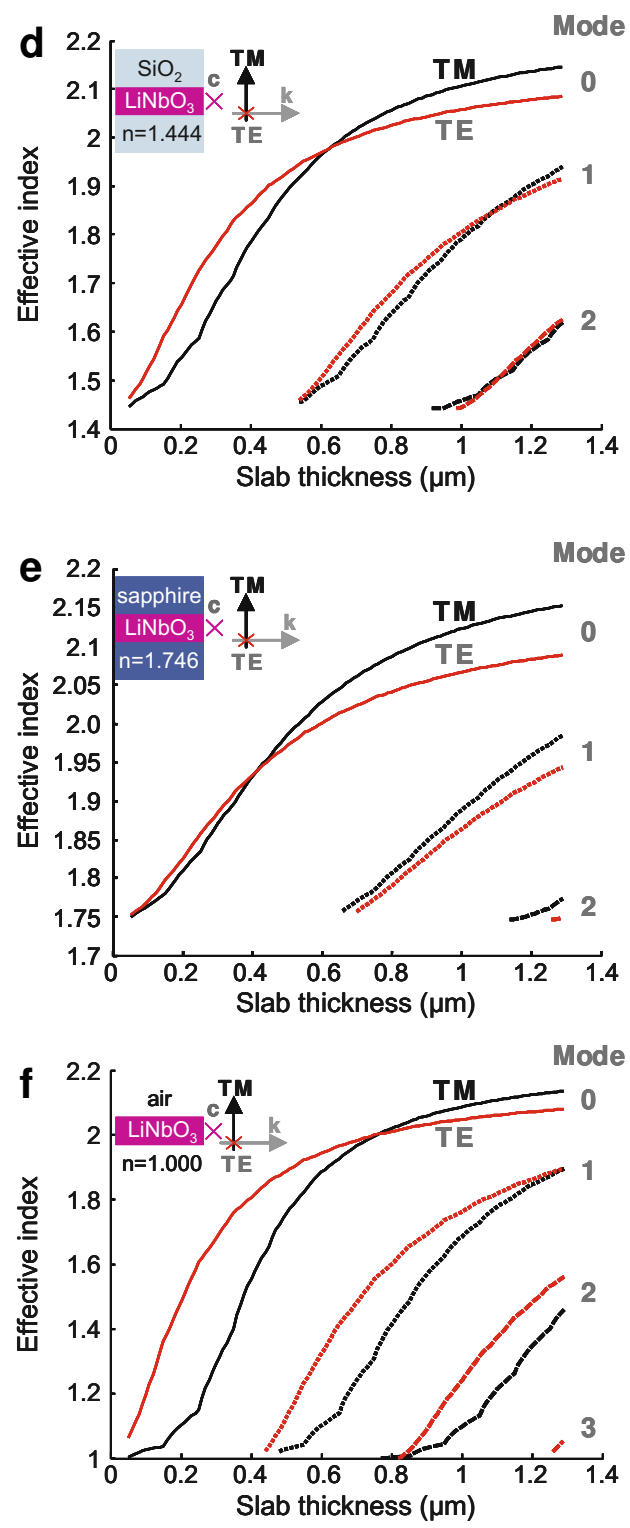

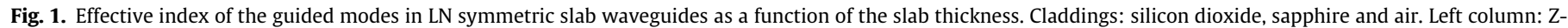
cut Y propagating LN slab waveguide; right column: X-cut Y propagating LN slab waveguide.

Table 1

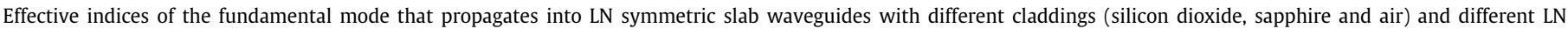
thicknesses. TE and TM polarizations are taken into account. Left column: Z-cut Y propagating LN slab waveguides; right column: X-cut Y propagating LN slab waveguides.

\begin{tabular}{|c|c|c|c|c|c|c|c|}
\hline \multirow[t]{2}{*}{ LN thickness $(\mu \mathrm{m})$} & & \multicolumn{3}{|l|}{ Z-cut } & \multicolumn{3}{|l|}{ X-cut } \\
\hline & & 0.2 & 0.6 & 1.0 & 0.2 & 0.6 & 1.0 \\
\hline \multirow[t]{2}{*}{$\mathrm{SiO}_{2}$ cladding } & TE polarization & 1.70 & 2.04 & 2.12 & 1.65 & 1.97 & 2.06 \\
\hline & TM polarization & 1.53 & 1.90 & 2.03 & 1.55 & 1.96 & 2.1 \\
\hline \multirow[t]{2}{*}{ Sapphire cladding } & TE polarization & 1.85 & 2.07 & 2.14 & 1.83 & 2.00 & 2.07 \\
\hline & TM polarization & 1.79 & 1.97 & 2.05 & 1.8 & 2.03 & 2.13 \\
\hline \multirow[t]{2}{*}{ Air cladding } & TE polarization & 1.53 & 2.02 & 2.12 & 1.48 & 1.94 & 2.05 \\
\hline & TM polarization & 1.09 & 1.83 & 2.02 & 1.1 & 1.88 & 2.08 \\
\hline
\end{tabular}


Table 2

Design parameters for the fabrication of TE/TM converter from LN symmetric slab waveguide.

\begin{tabular}{lll}
\hline & LN thickness $(\mathrm{nm})$ & Effective index \\
\hline $\mathrm{SiO}_{2}$ cladding & 625 & 1.96 \\
Sapphire cladding & 405 & 1.93 \\
Air cladding & 755 & 2.00 \\
\hline
\end{tabular}

and the width of the LN ridge) using the BeamPROP ${ }^{\mathrm{TM}}$ commercial software based on the beam propagation method [18]. The birefringence of LN is taken into account in all these simulations that are performed at $1.55 \mu \mathrm{m}$, one of the common telecom wavelengths.

The paper is subdivided as follow. First, the calculation method is described and is validated by comparing some results with those calculated by Rabiei et al. [17]. In the second part, we explore symmetric slab waveguides. Both TE and TM polarizations as well as Xand Z-cut LN layers are taken into account. We will show the possibility to find a suitable $\mathrm{LN}$ thickness that gives rise to the same effective index for both TE- and TM-polarized modes. As mentioned in a paper dealing with the coupling between TE and TM waves propagating into a thin film optical waveguide, two perpendicularly polarized modes having the same phase velocity could provide a complete exchange between the two waves [19]. Such geometry, in addition to the fabrication of interdigital electrodes, could lead to electro-optic TE/TM converters [20]. We will also discuss the coupling effects between slab and guided modes that cause losses. The last part of this article is devoted to LN asymmetric wire waveguides that allow a light confinement in the two directions. As a possible application, we finally determine the parameters of LN wire waveguides to fabricate a specific device: a TE/TM converter.

\section{Theoretical model: beam propagation method}

Since the goal of this work is to model different kinds of LN waveguides, the beam propagation method turns out to be the most convenient technique to compute the light propagation in slab waveguides. For this purpose, we use a commercial software, BeamPROP, that is a part of Rsoft Photonics Suite. This program is based on the finite difference beam propagation method (FD-BPM) and uses the Crank-Nicholson method [21]. The program consists of solving the scalar Helmholtz equation and uses transparent boundary conditions [22]. Polarization effects can be taken into account by introducing several vector beam propagation techniques [23]. High index-contrast and multimode propagation are possible by the use of the Pade-based wide-angle technique [24].

For each simulation, the multilayer structure is defined, taking into account the LN birefringence, the slab height and wire dimensions will be varied.

\section{Symmetric slab waveguides}

Numerical simulations are done at $1.55 \mu \mathrm{m}$. The first geometry consists of a thin LN film and two dielectric layers deposited on the upper and bottom sides of the LN serving as cladding of the waveguide. Silicon dioxide, sapphire and air, with refractive indices of $1.444,1.746$ and 1 , respectively, are chosen as claddings. For both Z-cut and X-cut Y propagating LN slab waveguides, the effective indices for both TE- and TM- polarized guided modes are calculated as a function of the slab thickness. The LN birefringence is taken into account. To summarize the parameters used for calculation, in X-cut (Z-cut respectively) $\mathrm{Y}$ propagating LN slab waveguides, the TE-polarized (TM-polarized respectively) mode is sensitive to the extraordinary refractive index $\left(n_{e}\right)$ of LN $\left(n_{e}=2.138 @ \lambda=1.55 \mu \mathrm{m}\right)$ while the TM-polarized (TE-polarized respectively) mode is sensitive to the ordinary refractive index of LN $\left(n_{o}\right)$ of LN $\left(n_{o}=2.210 @ \lambda=1.55 \mu \mathrm{m}\right)$. Fig. 1 shows the calculation results. The left column corresponds to Z-cut LN while the right column is devoted to X-cut LN.

Firstly, we validate our model by simulating the case published by Rabiei et al. [17]. Our model shows identical results than in [17].
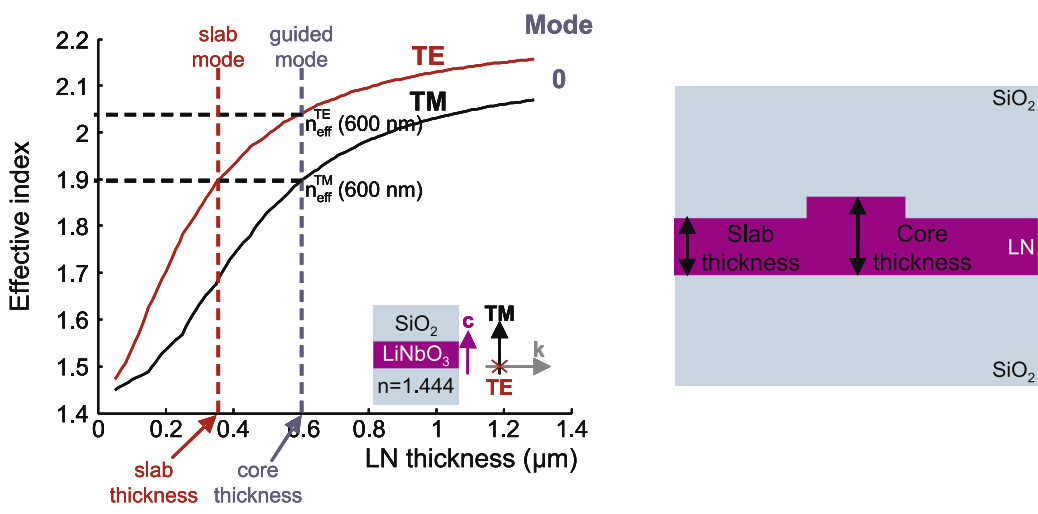

Fig. 2. Scheme of coupling effects between slab and guided modes in LN ridge waveguide.

Table 3

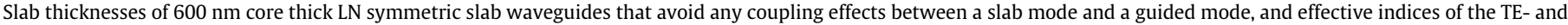
TM-polarized fundamental modes for different kinds of claddings and LN crystallographic orientations.

\begin{tabular}{|c|c|c|c|c|}
\hline & & $\mathrm{SiO}_{2}$ cladding & Sapphire cladding & Air cladding \\
\hline \multirow[t]{3}{*}{ Z-cut } & $n_{e f f}^{T M}(600 \mathrm{~nm})$ & 1.9 & 1.97 & 1.83 \\
\hline & $n_{\text {eff }}^{T E}(600 \mathrm{~nm})$ & 2.04 & 2.07 & 2.02 \\
\hline & Slab thickness $(\mathrm{nm})$ & 350 & 354 & 335 \\
\hline \multirow[t]{3}{*}{ X-cut } & $n_{e f f}^{T M}(600 \mathrm{~nm})$ & 1.96 & 2.03 & 1.88 \\
\hline & $n_{e f f}^{T E}(600 \mathrm{~nm})$ & 1.97 & 2.00 & 1.94 \\
\hline & Slab thickness (nm) & 585 & 534 & 492 \\
\hline
\end{tabular}


Secondly, we are interested in calculating the highest LN thickness (also called the cutoff thickness) that allows singlemode propagation. Independently of the LN crystallographic orientation and of the polarization, these cutoff thicknesses are $0.4,0.5$ and $0.6 \mu \mathrm{m}$ for air, $\mathrm{SiO}_{2}$, and sapphire claddings respectively. For thicker LN layers, the propagations become multimode. By comparing all these plots, it is also possible to find the structure that gives rise to the best mode confinement. For this purpose, let us chose three different LN thicknesses: $0.2 \mu \mathrm{m}$, that can be classed as a very thin layer where all types of waveguides are singlemode, $1 \mu \mathrm{m}$, which is already a thick layer, and $0.6 \mu \mathrm{m}$ which is a reasonable thickness for photonic crystal applications. Table 1 gives the effective indices of the TE- and TM-polarized fundamental guided modes that propagate in LN symmetric slab waveguides with the previously mentioned LN thicknesses and with different kinds of claddings ( silicon dioxide, sapphire and air). For each LN crystallographic orientation and thickness, and each cladding, bold prints exhibit the polarizations that give rise to the higher effective indices. Since the mode is more confined as effective index is closer to the LN refractive index, sapphire cladding is the most suitable for mode confinement while the worst confinement corresponds to a LN symmetric slab waveguide with an air cladding. This is in agreement with the fact that the LN thickness has to be thinner for air cladding than for sapphire cladding for singlemode waveguides. From Fig. 1 and Table 1 we can also see that, for Z-cut LN core, the TE-polarized mode is better confined than the TM-polarized mode independently of the LN thickness. This is not any more the case for the X-cut LN. An intersection point between the TMand TE-polarized fundamental mode plots occurs in Fig. 1. The LN thicknesses and the effective indices that correspond to these intersection points are reported in Table 2 for the three different claddings. Those design parameters could be suitable for the fabrication of TE/TM converters, since, at these intersection points, TMand TE-polarized fundamental modes propagate in the same manner insofar as the light sees the same effective indices. Among these three different TE/TM converters, the one corresponding to a LN suspended membrane (air/LN/air) has the higher effective index. With a LN thickness of $755 \mathrm{~nm}$, this air/LN/air slab waveguide is however multimode so that the entire energy of the light is not confined in the fundamental mode and will not be entirely suitable for TE/TM converter application. The sapphire cladding TE/TM converter is the one with the lower effective index but it is simultaneously the only one TE/TM converter that is singlemode. Since all the energy of the light keeps confined into the fundamental mode, sapphire/LN/sapphire waveguide appears to be the most efficient structure for TE/TM applications.

In symmetric waveguides, the light is confined in the slab plane. For some applications, like photonic crystal applications, it will be of great interest to get a confinement in the third direction. This is possible by creating a ridge in the LN layer as shown in Fig. 2. As previously mentioned by Rabiei et al. [17] for $\mathrm{SiO}_{2} / \mathrm{LN} / \mathrm{SiO}_{2}$ waveguides, in such structures some losses can arise from coupling effects between a guided mode and a slab mode if the effective index of the slab mode is higher than the effective index of the
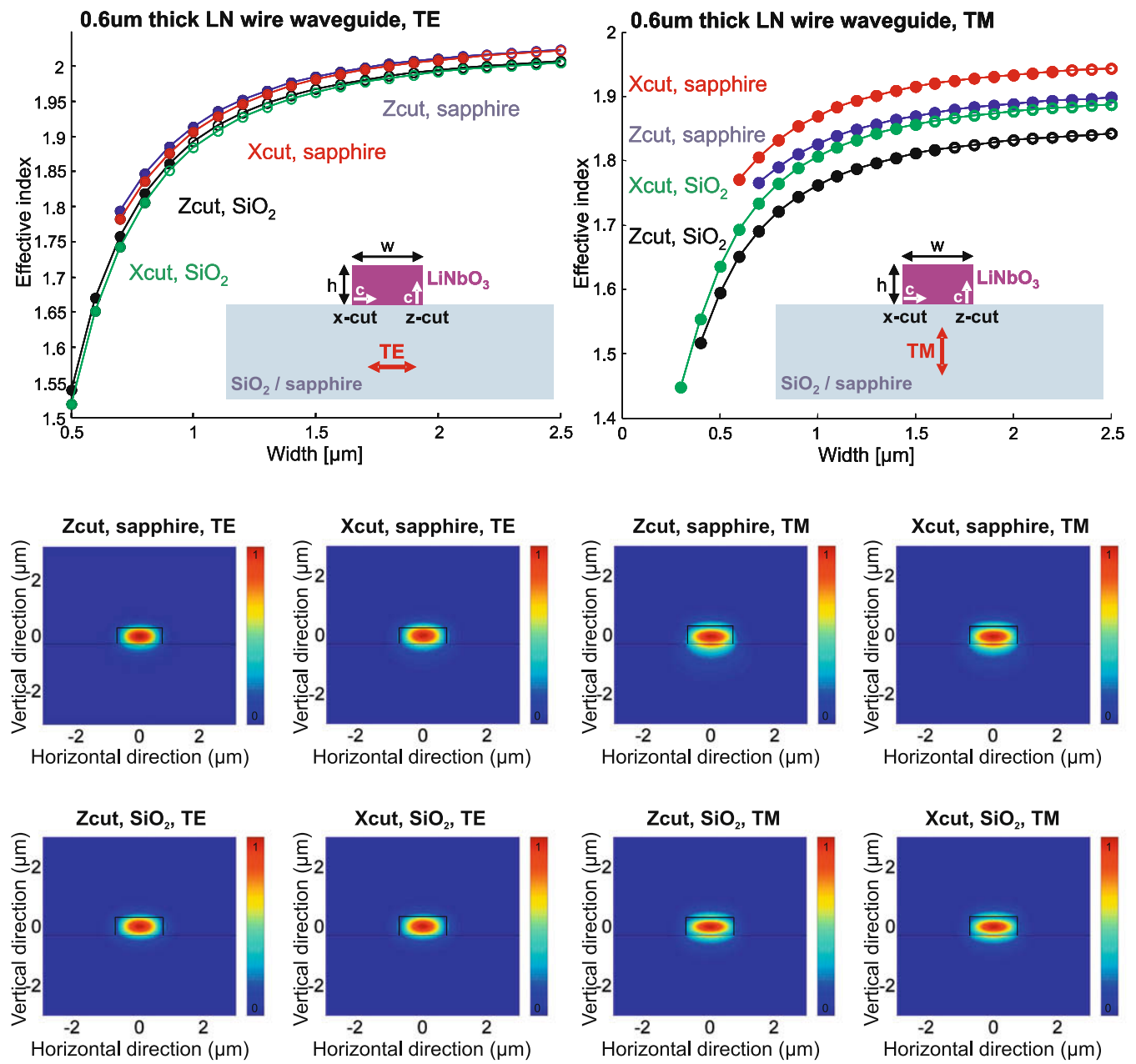

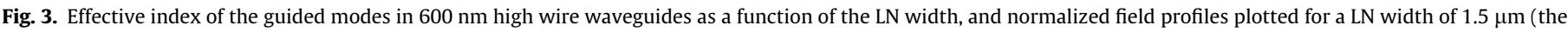
dark squares depict the ridge positions). 


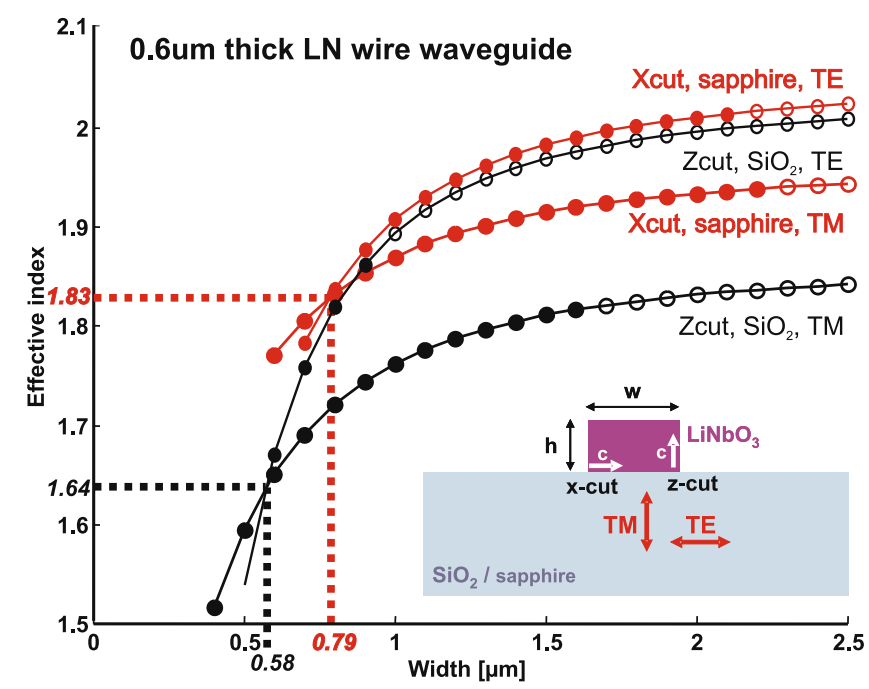

Fig. 4. Determination of the parameters for TE/TM converters in $\mathrm{LN}$ asymmetric wire waveguides.

Table 4

Design parameters for the fabrication of a TE/TM converter from LN asymmetric wire waveguide.

\begin{tabular}{lll}
\hline & LN wire width $(\mathrm{nm})$ & Effective index \\
\hline $\mathrm{SiO}_{2}$ substrate & 580 & 1.64 \\
Sapphire substrate & 790 & 1.83 \\
\hline
\end{tabular}

guided mode. In the following part, we will assess the minimal ridge height that will avoid any coupling effect for a whole LN core thickness of $600 \mathrm{~nm}$. This height was chosen because it corresponds to a LN thickness that can be drilled by FIB or RIE (reactive ion etching) without a lot of distorsion of the hole shape for photonic crystal applications and it is the highest thickness that allows a singlemode propagation in a symmetric slab waveguide with a sapphire cladding. In order to determine the ridge height that avoids any coupling effects, vertical lines corresponding to a $600 \mathrm{~nm}$ LN thickness are plotted as shown in Fig. 2. The ordinate coordinates that correspond to the intersection of each vertical line and the plots of the effective indices of the TE- and TM-polarized fundamental modes are reported in the Table 3. The slab thickness that avoids radiation losses is given by the abscissa coordinate from the intersection point between the horizontal line that goes through the smallest effective index and the curve of the mode polarized in the other direction. Those thicknesses are reported in Table 3. For Z-cut LN core, the effective index curve of the TM guided mode is located under the effective index curve of the TE mode independently of the claddings so that coupling effects between the TM-polarized mode and the TE-polarized slab mode occur. This is also the case for the X-cut LN symmetric slab waveguides with silicon dioxide and air claddings. For sapphire cladding, coupling effects occur between the TE-polarized guided mode and the TM-polarized slab mode.

The previous analysis results from calculations performed on slab structures and thus does not take into account the width ridge. This parameter is considered in the following.

\section{LN asymmetric wire waveguides}

In this part, we are interested in asymmetric waveguides composed of a $\mathrm{LN}$ ridge deposited on two different types of substrates: $\mathrm{SiO}_{2}$ and sapphire. Fig. 3 shows the plots of the effective indices of the fundamental mode that propagates in $600 \mathrm{~nm}$ thick LN asymmetric wire waveguides as a function of the LN width for both TE and TM polarizations and crystallographic orientations (X-cut and Z-cut LN). In these plots, where the symbol is filled, only the fundamental mode propagates, and where the symbol is open, there is at least a second mode at $1.55 \mu \mathrm{m}$ or more. Fig. 3 shows that the mode confinement strongly depends on the ridge width. For the TE polarization, the effective indices do not strongly vary with the crystallographic orientation of LN since X-cut LN and Zcut LN curves are close to each other for both types of cladding while for the TM polarization all curves are far from each other. By comparing all these curves, the best mode confinement corresponding to a singlemode propagation is reached for a Z-cut LN asymmetric wire waveguide with a $2.1 \mu \mathrm{m}$ width $\mathrm{LN}$. In this case, the fundamental mode is TE-polarized and its effective index is 2.15 .

As shown in Fig. 4, X-cut LN wire deposited on a sapphire substrate and Z-cut LN wire deposited on a silicon dioxide substrate allow the fabrication of TE/TM converters. The parameters of these TE/TM converters are summarized in Table 4. By comparing these parameters with those of symmetric slab waveguides (Table 3 ) it appears that the fundamental mode is more confined for TE/TM converters designed from symmetric slab waveguides. However, both TE/TM converters obtained from asymmetric wire waveguides allow singlemode propagation which is unfortunately not the case for the one which is fabricated from slab waveguide with a silicon dioxide cladding. We can conclude that the more efficient $\mathrm{TE} / \mathrm{TM}$ converters are symmetric slab waveguides with a sapphire cladding and LN wire deposited on a sapphire substrate.

\section{Conclusion}

This paper was devoted to the theoretical study of LN symmetric slab and asymmetric wire waveguides. The first kind of waveguide allows a light confinement in the slab plane while the light is confined in two directions in a LN asymmetric wire waveguide. The efficiency of LN waveguides with different geometries and claddings (air, sapphire and silicon dioxide) was discussed in term of light confinement, radiation losses and mode propagation from simulations based on the beam propagation method. We finally propose devices for TE/TM converter applications.

\section{Acknowledgement}

This work was supported by the European research project 3DDEMO STREP033297.

\section{References}

[1] K. Wong, Properties of Lithium Niobate, IEE, 2002.

[2] M. Levy, R. Osgood, R. Liu, L. Cross, G.C. Ill, A. Kumar, H. Bakhru, Appl. Phys. Lett. 73 (16) (1998) 2293.

[3] A. Radojevic, M. Levy, R. Osgood, A. Kumar, H. Bakhru, C. Tian, C. Evans, Appl. Phys. Lett. 74 (21) (1999) 3197.

[4] R. Roth, D. Djukic, Y. Lee, R. Osgood, S. Bakhur, B. Laulicht, K. Dunn, H. Bakhru, Appl. Phys. Lett. 89 (2006) 112906

[5] A. Radojevic, M. Levy, R. Osgood, D. Jundt, A. Kumar, H. Bakhru, Opt. Lett. 25 (14) (2000) 1034.

[6] A. Radojevic, M. Levy, R. Osgood, Appl. Phys. Lett. 73 (19) (1999) 2888

[7] T. Ramadan, M. Levy, R. Osgood, Appl. Phys. Lett. 76 (11) (2000) 1407.

[8] P. Rabiei, P. Gunter, Appl. Phys. Lett. 85 (20) (2004) 4603.

[9] R. Roth, T. Izuhara, R. Espinola, D. Djukic, R. Osgood, S. Bakhur, H. Bakhru, Opt Lett. 30 (9) (2005) 994.

[10] A. Radojevic, R. Osgood, M. Levy, A. Kumar, H. Bakhru, IEEE Photonics Technol Lett. 12 (12) (2000) 1653.

[11] P. Berini, G. Mattiussi, N. Lahoud, R. Charbonneau, Appl. Phys. Lett. 90 (2007) 061108.

[12] T. Izuhara, R. Roth, R. Osgood, S. Bakhur, H. Bakhru, Electron. Lett. 39 (15) (2003) 1118.

[13] D. Djukic, G. Cerda-Pons, R. Roth, R. Osgood, S. Bakhur, H. Bakhru, Appl. Phys. Lett. 90 (2007) 171116. 
[14] D. Ward, E. Statz, K. Nelson, R. Roth, R. Osgood, Appl. Phys. Lett. 86 (2005) 022908.

[15] M. Roussey, F.I. Baida, M.-P. Bernal, J. Opt. Soc. Am. B 24 (6) (2007) 1416.

[16] G. Burr, S. Diziain, M. Bernal, Opt. Express 16 (9) (2008) 6302.

[17] P. Rabiei, W. Steier, Appl. Phys. Lett. 86 (2005) 161115.

[18] R. Scarmozzino, A. Gopinath, R. Pregla, S. Helfert, J. Selected Top. Quantum Electron. 6 (2000) 150.
[19] T. P Sosnowski, G.D. Boyd, IEEE J. Quantum Elect. QE-10 (1974) 306.

[20] R. C Alferness, L.L. Buhl, Opt. Lett. 5 (1980) 473.

[21] R. Scarmozzino, R. Osgood, J. Opt. Soc. Am. A 8 (1991) 724.

[22] G. Hadley, Opt. Lett. 16 (1991) 624

[23] C. Xu, W. Huang, J. Chrostowski, S. Chaudhuri, J. Lightwave Technol. 12 (1994) 1926.

[24] G. Hadley, Opt. Lett. 17 (1992) 1743. 